\title{
Disorder-Assisted Exciton Transport
}

\author{
T. Scholak*, T. Zech, T. Wellens and A. Buchleitner \\ Physikalisches Institut der Albert-Ludwigs-Universität \\ Hermann-Herder-Str. 3, D-79104 Freiburg, Germany
}

\begin{abstract}
We discuss the possibly constructive role of disorder for the optimization of exciton transport in the FMO (Fenna-Matthews-Olson) light harvesting complex. Our analysis, which models the FMO as a 3D random graph, demonstrates the existence of a small fraction of optimal, though highly asymmetric, non-periodic conformations, which yield near-to-optimal coherent excitation transport. We argue that, on transient time scales, such quantum interference enhanced transport does always better than stochastic activation.
\end{abstract}

PACS: 87.15.hj, 05.60.Gg, 03.65.Ud, 03.65.Yz

\section{Introduction}

Quantum transport in "complex" systems is one of the key subjects dealt with in quantum chaos, at the heart, e.g., of mesoscopic and molecular physics, of the many implementations of quantum billiards, of decaying atomic systems, and of disordered systems, with applications from rogue waves and seismic patterns to microdisc lasers [1-7].

Recently, another class of complex systems, this time from the realm of biology, has been identified as a possible scenario for quantum coherence effects - the photosynthetic light harvesting complexes of algae and of higher plants: Energy quanta absorbed from incoming sunlight need to be transferred towards the photosynthetic reaction center (RC), where this energy is used to drive charge separation and fuel the plant's chemistry [8]. This transport occurs across a molecular network, with a stunning quantum efficiency (defined by the number of electronhole pairs created in the RC, per incoming photon) of more than $95 \%$ (quantum efficiency is not to be confused with energy efficiency!) $[9,10]$, on time scales of approximately $100 \mathrm{fs}$ [11]. The detailed transport mechanism is being elucidated only recently with the help of $2 \mathrm{D}$ spectroscopy, which, in some sense, is a refinement of simple pump-probe spectroscopy, and a nonlinear spectroscopic tool. It basically consists in reading out a four-wave mixing signal that probes the time dependence of some polarization inscribed into the medium [12]. The time evolution of this polarization pattern allows to map out the details of the exciton transfer process, and is typically represented by the time evolution of a two-dimensional Fourier transform of the time dependent four-wave mixing signal.

\footnotetext{
* Present address: Chemical Physics Theory Group, Department of Chemistry, University of Toronto, 80 St. George Str., Toronto, Ontario, M5S 3H6, Canada.
}

It is to be realized that these beautiful experiments attempt to monitor the quantum dynamics of truly complex, biochemical objects, with very complicated, multihierarchical and adaptable conformations, which, e.g. in the case of the Fenna-Mathews-Olson (FMO) complex, are of pretty disordered appearance (other complexes appear highly symmetric [13] — which suggests that nature has found many ways to achieve its purposes). This is precisely what makes these systems such a fascinating object for experimental and theoretical research: While their extraordinary performance in terms of functionality, robustness and adaptability clearly indicates that they incarnate specifically tuned solutions for the specific needs of the organism they are part of, their structural complexity goes far beyond what we used to deal with in experiments on fundamental quantum phenomena. Hence the wide-spread surprise as the above experiments sample ever more evidence that quantum coherence does prevail in these functional units over time scales that are relevant for their functionality, even at ambient temperature. This seems to be in contrast with what we believe to know about the fragility of quantum interference with respect to noise and/or disorder. Also let us note that all these experiments are performed on macroscopic samples of FMO complexes in liquid solution, which implies disorder averages, leading, e.g., to inhomogeneous broadening of the recorded signals [14], and thus rendering the clear and unambiguous identification of coherent transport mechanisms a rather involved experimental task.

In our attempt to reach an improved understanding of these systems, the following list of questions emerges very naturally:

- Where is the demarcation line between the system and the environment degrees of freedom? Indeed, the FMO is often modeled as a molecular network with each of its constituents equipped with an electronic two-level structure $[15,16]$. The number of 
constituents itself varies slightly (seven or eight) $[17,18]$, depending on the specific preparation of the sample. The electronic two-level structure provides the minimal model to establish dipole-dipole interaction mediated exciton transport across the network to the $\mathrm{RC}$, and is underpinned by advanced structure calculations gauged against spectroscopic data [19], but there is some debate about the role of background (e.g. vibrational) degrees of freedom [8]. Depending on your preferred point of view, and education, you may incorporate these additional degrees of freedom either into the system Hamiltonian, or into some strongly coupled part of the environment, which immediately implies some (hierarchical) environment structure. This, in turn, suggests non-Markovian environment coupling [20], and connects to the next question:

- What allows for the experimentally observed coherence times, at ambient temperature [21]? In other words, Markovian models of environment coupling of a network of two-level systems do not allow to reproduce these experimentally observed, unexpectedly long time scales [22]. This suggests that nature found a way to screen the quantum transport on the FMO against the broad band noise generated by the warm environment, at least on functionally relevant time scales. It is clear that any coherence must fade away on sufficiently long time scales, in an open, complex system. If nature indeed does employ quantum coherence for its (transport) purposes, then it had to tune the relevant time scales such as to get into target on the transient time scales on which noise has no time to act. One way to do so is by proper structuring of the environment, such as to slow down the detrimental influence of noise. Alternatively, and this brings us to the last question,

- Are there structural elements of the FMO network that enhance the transport efficiency? Nature may have tuned the FMO network structure itself, to achieve what quantum opticians call the "strong coupling limit" [23]: Make the relevant system dynamics faster than the time scale on which the environment interferes destructively. It is this latter line of thought which we will go after in the remainder of the present contribution.

Given the experimental evidence together with all the uncertainties sketched above on the detailed structure of the actual biochemical object under scrutiny, we want to accommodate all essential ingredients of the minimal model described above, though amended by a statistical component, in a sense inspired by the approach of random matrix theory [24]. The precise structure of the underlying Hamiltonian (of "system" and "environment" degrees of freedom, see above) being unknown to us, we might as well only fix some basic structural elements -

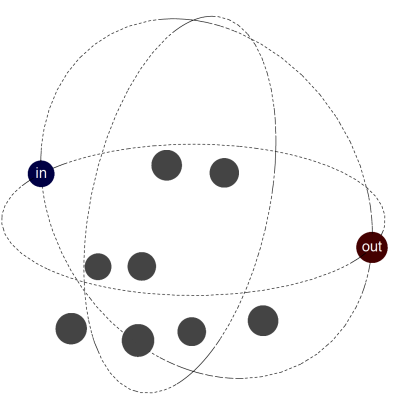

Fig. 1. Random graph model of the FMO complex, with $N=10$ molecular sites [25]. The exciton is injected at the site "in", and transferred to the RC once it reaches the site "out". In and out site are fixed at the poles of a sphere of diameter $d$, all other molecular sites are randomly placed within the sphere. Each site is equipped with an electronic two-level structure, thus mimicking a random, 3D array of spins that interact through dipole-dipole coupling, see Eq. (1). The coherent transport efficiency from "in" to "out" is optimized when all transition amplitudes interfere constructively.

which here will be the number of molecular constituents of the network, their individual electronic energy structure, their mutual interaction, and the position of two of them. We then investigate the statistics of the transport efficiency (to be defined further down) on a homogeneously distributed sample of molecular networks, under these constraints. We will first study transport efficiencies for the closed system, i.e. strictly unitary dynamics, and then open the system, by coupling each molecular site locally to a dephasing environment, and by coupling the output site to a sink (which represents the RC, where the exciton is to be delivered irreversibly).

\section{Model}

We model the FMO network as a fully connected, disordered finite graph with $N$ vertices, as depicted in Fig. 1. Coherent transport of a single excitation across a sample of molecular sites is generated by the Hamiltonian

$$
H=\sum_{i \neq j=1}^{N} v_{i, j} \sigma_{+}^{(j)} \sigma_{-}^{(i)},
$$

where $\sigma_{+}^{(j)}$ and $\sigma_{-}^{(i)}$ are mediate excitations and deexcitations of sites $j$ and $i$ from the local electronic ground state to the local excited state and vice versa, respectively. The excitation transfer $\sigma_{+}^{(j)} \sigma_{-}^{(i)}$ from site $i$ to site $j$ has a strength $v_{i, j}=v_{j, i}$ which depends on the specific nature of the inter-site coupling - that we assume to be of resonant (isotropic) dipole type, $v_{i, j}=\alpha / r_{i, j}^{3}$, with $r_{i, j}=\left|\boldsymbol{r}_{i}-\boldsymbol{r}_{j}\right|$ and $\boldsymbol{r}_{j}$ the position vectors of individual sites. Input and output site define the poles of a sphere of diameter $d$ which, via the coupling constant $v_{\text {in,out }}=\alpha / d^{3}$, sets the natural time scale of the dynamics induced by $H$. The positions of the remaining molecular sites are randomly (uniformly) chosen within this sphere, 
which induces a random distribution of the remaining $v_{i, j}$. Since additional sites between input and output contribute to additional transition amplitudes, they tend to render the transport more efficient, in some analogy, e.g., to multiphoton excitation and ionization processes of atomic or molecular Rydberg states [26], or to the momentum spread of ultracold atoms under periodic forcing [27].

Next, we need to specify what we understand by "transport efficiency". In colloquial terms, "efficient" means "a lot", and "fast". In our picture, this implies that an excitation initially localized at the input site is to be transferred, ideally, with probability one to the output site. "Fast" requires a benchmark, and this is naturally given by the intrinsic time scale defined by the direct coupling between input and output site, $\pi / 2\left|v_{\text {in,out }}\right|$. It is therefore suggestive to use [28]:

$$
\mathcal{P}=\max _{t \in[0, \mathcal{T}]} \mid\left.\langle\text { out } \mid \psi(t)\rangle\right|^{2}, \quad \mathcal{T}=0.1 \times \pi / 2\left|v_{\text {in }, \text { out }}\right|
$$

as our figure of merit for the transport efficiency. Let us note that this choice is not completely unambiguous - it rather needs to be understood in the context of the specific physical question being asked. Our above definition focuses on the short time dynamics and thus probes the enhancement of the effective coherent coupling between input and output site through the intermediate sites. However, in the closed system, strong effective coupling between input and output may lead to rapid (coherent) oscillations of the exciton between the opposite poles of the sphere, and if the excitation is to be absorbed at the output site with a certain drain rate $\Gamma$, the coherent oscillation frequency needs to roughly match the drain rate, for rapid excitation extraction towards the $\mathrm{RC}$, and therefore must not be too fast either*! Nonetheless, since the key issue raised by the above-mentioned experiments is the potential optimization of exciton transport by quantum coherence, such quantum advantage can only stem from transient time scales (as clearly spelled out by experimental data, which show that quantum beats only persist for short times, and not asymptotically). It is for this very reason that we choose the above definition of efficiency for our present discussion, but will come back to this issue further down.

\section{Results}

Figure $2 \mathrm{a}$ shows the variation of the transport efficiency $\mathcal{P}$ for a sample of 500 different random distributions of $N=7$ sites as in the FMO complex [29]. Let us note that the abscissa does not represent a continuous parameter here, but rather the number of the random configuration generated in our statistical sam-

\footnotetext{
* To optimize the extraction, nature may obviously tune the effective coupling as well as the drain rate. However, it is not a priori obvious whether extraction of the exciton in one chunk is preferable to its slow leaking out, upon integration over several coherent oscillations.
}

pling, such as to highlight the high sensitivity of $\mathcal{P}$ under variation of the sample's geometry. Figure $2 \mathrm{~b}$ shows the associated probability density $f_{\mathcal{P}}$ of $\mathcal{P}$, sampled over $2.5 \times 10^{8}$ random configurations of $N=7$ molecular sites. Clearly, the vast majority of configurations is found to be inefficient, giving rise to a rather low mean value $\overline{\mathcal{P}}=\int_{0}^{1} x f_{\mathcal{P}}(x) \mathrm{d} x \approx 4.85 \%$. However, a small fraction of configurations - one out of $2 \times 10^{5}$ - exhibits $\mathcal{P}>90 \%$, due to constructive multipath interference upon transmission. The general picture emerging here is strongly reminiscent of conductance fluctuations as a generic feature of quantum transport in disordered media [30], with the peculiarity that we are here dealing with a very finite system composed of not more than ten sites (and an effective Hilbert space dimension $N$, if only one excitation is transported at a time), i.e. that the quantum transport will be clearly dominated by finite size effects, and, hence, by strong fluctuations. This also implies a caveat with respect to the frequently used argument that (Anderson-like) localization effects will block transport in this kind of molecular networks [15, 16], as a consequence of destructive interference, and that, therefore, noise is required to disrupt this quantum interference, such as to restore transport. While this argument does indeed hold for large samples, which are somewhat close to the thermodynamic limit, it neglects the dominant role of fluctuations in the limit of small samples as we are here dealing with, and as nicely spelled out by Fig. 2 a.

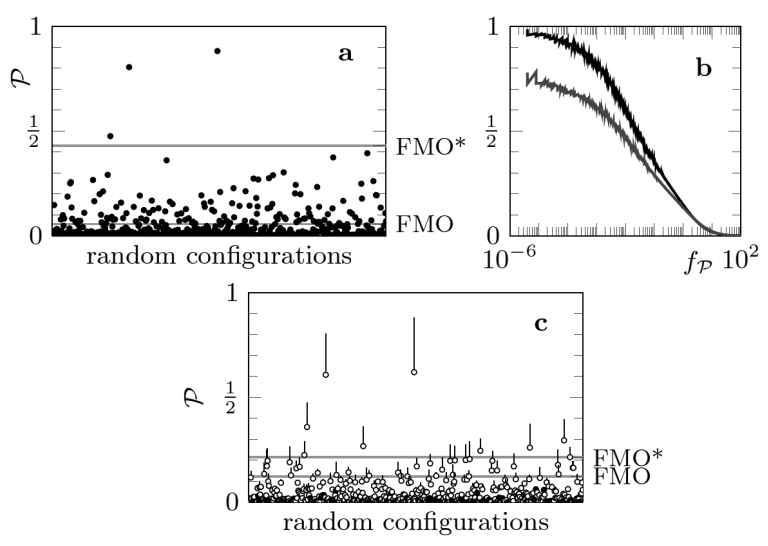

Fig. 2. (a) Fluctuation of the transfer efficiency $\mathcal{P}$ from input to output, for 500 different random conformations of $N=7$ sites (dots) [28]. Horizontal gray lines indicate the transfer efficiency of the experimentally infered [19] FMO Hamiltonian, as well as that of the optimal configuration FMO* $^{*}$ which is compatible with the experimental error margin. (b) Probability densities $P(\mathcal{P})$ of the transfer efficiency $\mathcal{P}$ for $2.5 \times 10^{8}$ different conformations. For fully coherent dynamics (black curve) the mean value of $p_{\text {out }}^{(\mathcal{T})}$ amounts to $4.9 \%$, and only 4.5 out of million configurations provide efficiencies larger than $90 \%$. In the presence of local dephasing (gray line) the mean efficiency drops to $3.9 \%$. (c) Gains (closed dots) and losses (open dots) of the transfer efficiency with dephasing. 

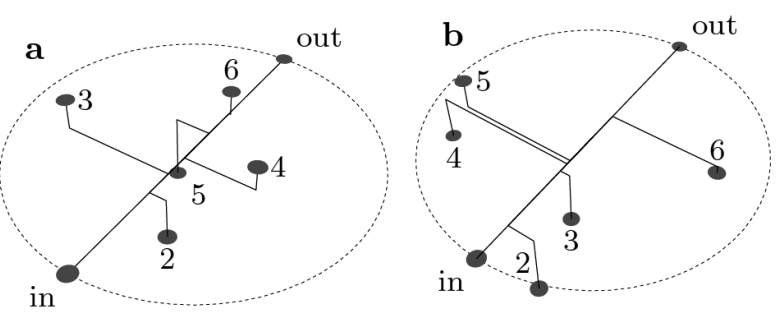

Fig. 3. Comparison of molecular conformations with (a) optimal vs. (b) generic transfer efficiencies $\mathcal{P}$. The conformation in (a) was optimized with the help of a genetic algorithm. Neither one of both conformations has any distinctive symmetry or periodicity properties which would allow to infer its transport properties.

It is precisely the rare, optimal configurations which provide the enhanced quantum transport as identified in Fig. 2 we are looking for, and it is immediate to ask for their specific properties. Figure 3 compares (a) an optimal configuration which, indeed, generates $\mathcal{P}=100 \%$, after optimization by a genetic algorithm, with (b) a generic configuration with $\mathcal{P}=4.8 \%$. There is no obvious, qualitative difference which allows to distinguish one configuration from the other. It is indeed easy to see that a necessary condition for unit transfer efficiency is the existence of at least one eigenstate of $H$ which has equal overlap with the input state (exciton localized at "in") and the output state (exciton localized at "out"). However, how such spectral property correlates with the geometry of the molecular network remains an open question, possibly adequately dealt with in the framework of random vector statistics [31].

What is the potential relevance of the above observations for the actual transport problem in the FMO complex?

(1) The FMO complex is embedded in a noisy environment, and it therefore needs to be established whether the transport efficiency of the above, "optimal" conformations prevails in the presence of noise. Indeed, Fig. 2c shows that this is the case. It compares the transport efficiencies of the same configurations as in Fig. 2a in the presence of (locally, for each molecular site in contact with its own, private environment) dephasing noise to those in its absence. The underlying dynamics is here modeled by the simplest possible model for open system evolution, incarnated by the master equation (where we set $\hbar=1$, for convenience)

$$
\dot{\varrho}(t)=-\mathrm{i}[H, \varrho(t)]-4 \gamma \sum_{i \neq j=1}|i\rangle\langle i|\varrho| j\rangle\langle j| .
$$

Obviously, noise will disrupt destructive as well as constructive interference effects, and the "optimal" conformations' performances will therefore suffer, while the blocking conformations' (those with low efficiencies, in the absence of noise) will gain. However, on the transient time scales which we here focus on (and which are the only relevant time scales, if one is interested in the impact of quantum interference effects on the transport process, provided one does not invoke long-time memory effects in the environment - tantamount of slow background modes), optimal conformations remain optimal, and inefficient ones remain inefficient. Even in the presence of noise, a clear dichotomy in conformation space (of which we are still unable to identify the distinctive features) persists.

(2) There are very advanced, state of the art structure calculations [19], which, combined with experimental data, allow to infer some effective Hamiltonian which is often used for dynamical simulations of the FMO quantum dynamics. Such simulations show that this best, effective FMO Hamiltonian yields coherent transport efficiencies of modest $5.7 \%$. However, this is not in conflict with our above, statistical approach, since the error bars that garnish the effective Hamiltonian are compatible with optimized conformations that yield transport efficiencies of up to $43.1 \%$ (see the efficiencies FMO vs. FMO* in Fig. 2a, c) [28].

(3) There is evidence [14] that the microscopic conformation of different FMO complexes in the same organism has considerable variability, hence there appears to be some unavoidable random element which may speak against a deterministic "engineering" of optimal conformations by nature. On the other hand, this might be too biased an engineer's point of view. It appears very clear that many if not most biological processes are triggered by stochastic processes (irrespective of classical or quantum) [32, 33], and that most biological functional units are multifunctional (and, therefore, are not optimized for one single purpose - it suffices to be better). The distribution of molecular conformations itself can be considered as a stochastic process, and we speculate that it may suffice that just a small but finite fraction of them exhibits near-to-optimal efficiency. If the constraints defined by the FMO macromolecular environment induce statistics of the transfer efficiencies such that in any sufficiently large sample of FMO's there is a sufficiently large fraction of efficient ones, the biological purpose is met, and in a very robust manner. It would not be too surprising if the evolutionary advantage would be encoded in the tail of the distribution.

(4) In the actual FMO transport problem, the excitation is to be delivered irreversibly to the RC, on output, which can be modeled by locally adding a decay term to the output site. This is implemented by adding a term

$$
\left.L_{\operatorname{sink}}(\varrho)=\Gamma\left(|0\rangle\langle\text { out }|\varrho| \text { out }\rangle\langle 0|-\frac{1}{2}\{\mid \text { out }\rangle\langle\text { out }|, \varrho\right\}\right),
$$

to the right hand side of Eq. (3), where $|0\rangle$ and $\{$,$\} are$ the ground state of the molecular network and an anticommutator, respectively. The, thus implied, change of boundary conditions with respect to the closed system considered above also changes the geometry of those conformations which provide optimal transport efficiency, provided the sink coupling $\Gamma$ is not too small [34]. How- 
ever, the addition of a sink does not change the fundamental picture. Optimal transport efficiencies are achieved by those networks which define optimal coherent coupling between input and output site, and such coherence-induced optimal transport does always better than noise-induced excitation transfer. This is illustrated in Fig. 4 by a plot of the probability density of the excitation transfer time

$$
\mathcal{T}=\Gamma \int_{0}^{\infty} t\langle\text { out }|\varrho(t)| \text { out }\rangle \mathrm{d} t
$$

(which is an alternative efficiency quantifier when the time scale for irreversible delivery of the excitation to the $\mathrm{RC}$ is to be quantified), as a function of the dephasing rate $\gamma$. Furthermore, since noise disrupts the systems' specific interference properties, we see that it also blurs the distinction between different conformations, and thus excludes conformational properties as potential criteria for evolutionary selection.

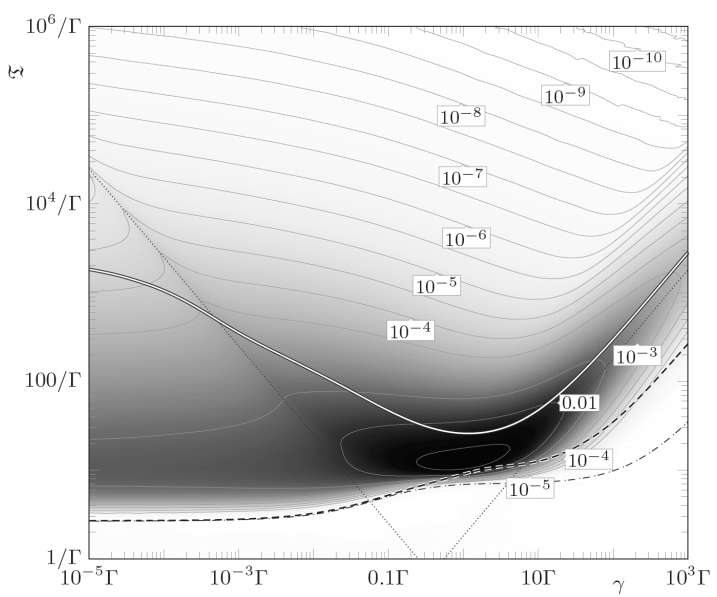

Fig. 4. Probability density $f_{\mathcal{T}}$ of the average excitation transfer time $\mathcal{T}$, Eq. (5), for $N=7$ molecular sites and sink rate $\Gamma=10 / T$, as a function of the dephasing rate $\gamma[35]$. The two dotted, diagonal lines are given by the dephasing time $\mathcal{T}_{\text {deph }}=(4 \gamma)^{-1}$, and by an approximate Zeno time $\mathcal{T}_{\text {Zeno }} \propto \gamma$ (beyond which transport is "frozen", due to the quantum Zeno projection mechanism), respectively. On time scales $\mathcal{T}>\mathcal{T}_{\text {deph }}$, the purity of the excitonic state on the molecular network has dropped to its minimum value, hence the transport is essentially classical. The white line shows the median $\tilde{\mathcal{T}}$, the dash-dotted line the minimum transfer time, and the dashed line the transfer time of a configuration that has been optimized for $\gamma=0$. Clearly, this latter conformation achieves optimal transfer times over a broad range of dephasing rates, shorter than the dephasing time, and in particular shorter than the optimal noise induced transfer times - associated with the highest densities in the plot, above the dephasing time. This observation is fully consistent with our comparison of transfer efficiencies on short time scales, in Fig. 2 above.

\section{Conclusions}

We have shown that optimal excitation transport in the FMO complex may be due to the finite probability of conformations that lend constructive multi-path interference from input to output. Since such large scale excursions of the transfer efficiency for given sample size can be considered as rare events, this problem appears an attractive object for appropriate statistics [36]. In particular, the scaling of the probability of such rare events with the size of the network is one of the important questions ahead, since this might give a hint of what determines the optimal size of FMO like networks in nature.

\section{Acknowledgments}

Enjoyable and instructive discussions with Roberto Mulet, Fernando de Melo, Florian Mintert and Markus Tiersch, as well as financial support by the German National Academic Foundation (T.Z.) are gratefully acknowledged.

\section{References}

[1] J. Madroñero, A. Ponomarev, A.R.R. Carvalho, S. Wimberger, C. Viviescas, A. Kolovsky, K. Hornbeger, P. Schlagheck, A. Krug, A. Buchleitner, Adv. At. Mol. Opt. Phys. 53, 33 (2006).

[2] M. Lawniczak, S. Bauch, O. Hul, L. Sirko, Phys. Rev. E 81, 046204 (2010).

[3] R. Höhmann, U. Kuhl, H.-J. Stöckmann, L. Kaplan, E.J. Heller, Phys. Rev. Lett. 104, 093901 (2010).

[4] M. Hartung, T. Wellens, C.A. Müller, K. Richter, P. Schlagheck, Phys. Rev. Lett. 101, 020603 (2008).

[5] M.G. Brown, J.A. Colosi, S. Tomsovic, A.L. Virovlyansky, M.A. Wolfson, G.M. Zaslavsky, J. Acoust. Soc. Am. 113, 533 (2003).

[6] C. Lomnitz, J. Flores, O. Novaro, T.H. Seligman, R. Esquivel, Bull. Seism. Soc. Am. 89, 14 (1999).

[7] Q.H. Song, L. Ge, A.D. Stone, H. Cao, J. Wiersig, J.B. Shim, J. Unterhinninghofen, W. Fang, G.S. Solomon, Phys. Rev. Lett. 105, 103902 (2010).

[8] G.R. Fleming, G.D. Scholes, Y.-C. Cheng, Procedia Chem. 3, 38 (2011).

[9] R.K. Chain, D.I. Arnon, Proc. Natl. Acad. Sci. USA 74, 3377 (1977).

[10] R.E. Blankenship, D.M. Tiede, J. Barber, G.W. Brudvig, G. Fleming, M. Ghirardi, M.R. Gunner, W. Junge, D.M. Kramer, A. Melis, T.A. Moore, C.C. Moser, D.G. Nocera, A.J. Nozik, D.R. Ort, W.W. Parson, R.C. Prince, R.T. Sayre, Science 332, 805 (2011).

[11] S.E. Bradforth, R. Jimenez, F. van Mourik, R. van Grondelle, G.R. Fleming, J. Phys. Chem. 99, 16179 (1995).

[12] S. Mukamel, D. Abramavicius, L. Yang, W. Zhuang, I.V. Schweigert, D.V. Voronine, Acc. Chem. Res. 42, 553 (2009).

[13] R.E. Blankenship, Molecular Mechanisms of Photosynthesis, 1st ed., Blackwell Publ. Ltd., 2001.

[14] A. Ishizaki, G.R. Fleming, J. Phys. Chem. B $\mathbf{1 1 5}$ 6227 (2011) 
[15] P. Rebentrost, M. Mohseni, I. Kassal, S. Lloyd, A. Aspuru-Guzik, New J. Phys. 11, 033001 (2009).

[16] F. Caruso, A.W. Chin, A. Datta, S.F. Huelga, M.B. Plenio, J. Chem. Phys. 131, 105106 (2009).

[17] D. Tronrud, J. Wen, L. Gay, R. Blankenship, Photosynthesis Res. 100, 79 (2009).

[18] C. Olbrich, J. Strümpfer, K. Schulten, U. Kleinekathöfer, J. Phys. Chem. B 115, 758 (2011).

[19] J. Adolphs, T. Renger, Biophys. J. 91, 2778 (2006).

[20] K.H. Hughes, C.D. Christ, I. Burghardt, Chem. Phys. 131, 124108 (2009).

[21] G. Panichayangkoon, D. Hayes, K.A. Fransted, J.R. Caram, E. Harel, J. Wen, R.E. Blankenship, G.S. Engel, Proc. Natl. Acad. Sci. USA 107, 12766 (2010).

[22] M. Sarovar, A. Ishizaki, G.R. Fleming, K.B. Whaley, Nat. Phys. 6, 462 (2010).

[23] F. de Melo, A. Buchleitner, Lect. Notes Phys. 808, 253 (2010).

[24] F. Haake, Quantum Signatures of Chaos, Springer, Berlin 2001.

[25] T. Scholak, F. Mintert, T. Wellens, A. Buchleitner, Semicond. Semimet. 83, 1 (2010).

[26] R. Bümel, A. Buchleitner, R. Graham, L. Sirko, U. Smilansky, H. Walther, Phys. Rev. A 44, 4521 (1991).
[27] V. Milner, D.A. Steck, W.H. Oskay, M. Raizen, Phys. Rev. E 61, 7223 (2000).

[28] T. Scholak, F. de Melo, T. Wellens, F. Mintert, A. Buchleitner, Phys. Rev. E 83, 021912 (2011).

[29] Y.-C. Cheng, G.R. Fleming, Ann. Rev. Phys. Chem. 60, 241 (2009).

[30] B. Kramer, A. MacKinnon, Rep. Prog. Phys. 56, 1469 (1993).

[31] F. Haake, K. Życzkowski, Phys. Rev. A 42, 1013 (1990).

[32] P. Hänggi, P. Talkner, M. Borkovec, Rev. Mod. Phys. 62, 251 (1990).

[33] T. Wellens, V. Shatokhin, A. Buchleitner, Rep. Prog. Phys. 67, 45 (2004).

[34] T. Scholak, T. Wellens, A. Buchleitner, J. Phys. B 44, 184012 (2011).

[35] T. Scholak, T. Wellens, A. Buchleitner, Europhys. Lett. 96, 10001 (2011).

[36] A. Lakshminarayan, S. Tomsovic, O. Bohigas, S.N. Majumdar, Phys. Rev. Lett. 100, 044103 (2008). 